Abstracta Iranica Abstracta Iranica

Revue bibliographique pour le domaine irano-aryen

Volume 40-41 | 2019

Comptes rendus des publications de 2017-2018

\title{
Marie Favereau (éd.). La Horde d'Or et l'islamisation des steppes eurasiatiques/The Golden Horde and the Islamisation of the Eurasian Steppes
}

\section{Simon Berger}

\author{
(2) OpenEdition \\ Journals \\ Édition électronique \\ URL : http://journals.openedition.org/abstractairanica/50427 \\ DOI : 10.4000/abstractairanica.50427 \\ ISBN : 1961-960X \\ ISSN : 1961-960X \\ Éditeur : \\ CNRS (UMR 7528 Mondes iraniens et indiens), Éditions de l'IFRI
}

Référence électronique

Simon Berger, «Marie Favereau (éd.). La Horde d'Or et l'islamisation des steppes eurasiatiques/The Golden Horde and the Islamisation of the Eurasian Steppes ", Abstracta Iranica [En ligne], Volume 40-41 I 2019, document 18, mis en ligne le 30 décembre 2019, consulté le 17 avril 2021. URL : http:// journals.openedition.org/abstractairanica/50427 ; DOI : https://doi.org/10.4000/abstractairanica. 50427

Ce document a été généré automatiquement le 17 avril 2021.

Tous droits réservés 


\title{
Marie Favereau (éd.). La Horde d'Or et l'islamisation des steppes eurasiatiques/ The Golden Horde and the Islamisation of the Eurasian Steppes
}

\author{
Simon Berger
}

\section{RÉFÉRENCE}

Marie Favereau (éd.). La Horde d'Or et l'islamisation des steppes eurasiatiques/The Golden Horde and the Islamisation of the Eurasian Steppes. Revue des mondes musulmans et de la Méditerranée vol. 143, Aix-en-Provence : Presse Universitaire de Provence, 2018

1 Ce numéro de la Revue des mondes musulmans et de la Méditerranée prend pour thème la Horde d'Or, qui constituait l'un des quatre grands khanats issus de l'empire mongol unitaire, disloqué en 1259, et qui s'étendait sur les steppes du Nord-Ouest de l'Eurasie. Il en traite sous l'angle de l'islamisation, l'un des phénomènes majeurs de l'empire mongol dans ses parties centrales et occidentales, et l'un de ses héritages les plus durables, et la filiation entre les études contenues dans ce volume avec l'œuvre pionnière en la matière de Devin DeWeese (Islamization and Native Religion in the Golden Horde, Baba Tükels and Conversion to Islam in Historical and Epic Tradition, University Park, 1994) est affirmée dès l'introduction par Marie Favereau. Le dossier comprend quatorze contributions, réparties en quatre parties: «Tolerance as a Political Tool», "Indigenous Islam », « Channels of Islamisation», « The Horde Islamic Legacies », plus une annexe : « New Sources in Translation. Si les chercheurs travaillant en premier lieu sur les textes sont majoritaires, l'archéologie et l'histoire de l'art sont également représentées.

2 Dans le premier article, Yihao Qiu («Independent Ruler, Indefinable Role. Understanding the History of the Golden Horde from the Perspectives of the Yuan 
Dynasty », p. 29-48) démontre que les conflits armés qui opposent le souverain de la Horde d'Or, Berke, à celui de l'Ilkhanat, Hülagu, et au Grand Khan Qubilai, n'ont pas pour cause la conversion à l'islam du premier, mais s'inscrivent dans la rivalité ancrée dans le temps entre lignages jochides et toluides. Les deux articles suivant ont en commun d'utiliser les sources franciscaines sur la Horde d'Or. Thomas Tanase (« A Christian Khan of the Golden Horde? 'Coktoganus' and the geopolitics of the Golden Horde at the time of its Islamisation », p. 49-63) entend mettre en avant l'existence à la cour de la Horde d'Or d'un parti chrétien qui aurait compté un prétendant au trône après la mort du khan Toqta (1290-1312), « Coktoganus », ou Kutukan. Roman Hautala ("Comparing the Islamisation of the Jochid and Hülegüid Uluses: Muslim and Christian Perspectives », p. 65-79) montre que les conversions des souverains mongols n'étaient pas suivies de conversions massives de leurs sujets, et que les politiques de conversions forcées furent toujours de courte durée et se heurtèrent à de fortes résistances de la part des nomades, aussi bien dans la Horde d'Or que dans l'Ilkhanat d'Iran.

3 Irina Shingiray (« An Islamicate Body: A Case Study of a Nomadic Burial from the Core Territory of the Golden Horde », p. 83-105) prend ensuite le cas d'une tombe du XIII ${ }^{\text {e }}$ $\mathrm{XIV}^{\mathrm{e}}$ siècle en milieu nomade pour mettre en avant l'importance de l'islam dans la vie quotidienne des populations nomades des steppes, par-delà la cour et les milieux urbains. Devin DeWeese (« A Khwārazmian Saint in the Golden Horde: Közlük Ata [Gözlī Ata] and the Social Vectors of Islamisation », p. 107-132) étudie le cas de Közlük Ata, ancêtre putatif de la tribu sainte des Turkmènes Ata, souligner la dimension essentiellement sociale de l'islamisation et pour montrer le rôle de l'islam comme ciment social de nouvelles communautés politiques au XIVe siècle. Dans le dernier article de cette action, Zvezdana Dode ("The Golden Tent Paradigm: Between the Mongols and Islam », p. 133-147) analyse le motif de la tente d'or du souverain dans les sources persanes.

4 Au sein de la partie suivante, Andrew Peacock (« Islamisation in the Golden Horde and Anatolia: Some remarks on travelling scholars and texts », p. 151-163) et Nicole KançalFerrari ("Contextualising the Decorum of Golden Horde-Period Mosques in Crimea: Artistic Interactions as Reflected in Patronage and Material Culture», p. 191-213) s'attachent tous deux à montrer les liens étroits existant entre la Horde d'Or et l'Anatolie seljūqide et ilkhanide, le premier à travers la circulation des savoirs et de savants, la seconde par le style architectural des monuments mongols construits en Crimée, qu'elle rapproche de ceux d'Anatolie et de Transcaucasie construites à la même période. Entre ces deux contributions deux articles, celui de Marie Favereau ("Tarkhan: A Nomad Institution in an Islamic Context», p. 169-185) analyse l'institution nomade du tarkhanlï, par lequel les dirigeants mongols conféraient à un individu ou à un groupe un statut particulier, associé à divers privilèges, dont l'exemption fiscale et le libre accès à la personne du souverain. Ishayahu Landa (« From Mongolia to Khwārazm: The Qonggirad Migrations in the Jochid Ulus (13th.-15th. c.) ", p. 215-231) explore ensuite le rôle des membres du lignage Qonggirad au sein de l'ulus jochide, et la manière dont une aristocratie qonggirad sut maintenir un pouvoir autonome local au Khẉārazm à la fin du XIV et au début du XV siècle.

Dans la dernière section, Vadim Trepavlov (" The Takht Eli Khanate: The State System at the Twilight of the Golden Horde ", p. 235-247) aborde l'héritage de la Horde d'Or au sein du khanat de Takht Eli, dit aussi la Grande Horde, au XV siècle. Enfin, Maya Petrovich ( Uncanny Beloveds and the Return of the Repressed: Ottoman Encounters 
with the Qipchaq Steppe ", p. 249-263) explore le statut ambivalent des Tatars de Crimée et de la Volga dans la littérature et au sein des élites ottomanes.

6 Le dossier se conclut par une annexe sur les sources : Michel Balivet et Homa LessanPezechki (« Seldjukides de Rūm contre Horde d'Or : l'expédition de Crimée d'après Ibn Bībī [vers 1225] », p. 267-283) donnent ici à lire un nouvel extrait traduit du Mukhtașar-i Saljuqnāma d'Ibn Bībī (dont une large partie avait déjà été publiée dans Michel Balivet, Homa Lessan-Pezechki et René Mounier, Les Turcs seldjoukides d'Anatolie XIe-XIVe siècle. Une anthologie des sources premières, vol. I : Ibn Bîbî, Aix-en-Provence, 2017). Milyausha Shamsimukhametova présente Qalandarnāma, œuvre du soufi et savant anatolien Abū Bakr Qalandar Rūmī rédigée au sein du khanat de la Horde d'Or au XIV ${ }^{\mathrm{e}}$ siècle. Deux cartes, non exemptes d'erreurs, situées en introduction, complètent le dossier. Celui-ci relève au final d'un effort remarquable salutaire pour mettre en lumière la Horde d'Or, trop souvent délaissée par les historiens au profit de l'Ilkhanat ou de la Chine des Yuan, au sein de l'historiographie de l'empire mongole.

\section{AUTEURS}

\section{SIMON BERGER}

Doctorant EHESS, CETOBAC, Paris 